\title{
Medium-sized Satellites of Large Kuiper Belt Objects
}

\author{
Michael E. Brown ${ }^{1}$ (i) and Bryan J. Butler ${ }^{2}$ \\ ${ }^{1}$ California Institute of Technology, Pasadena, CA 91125, USA \\ ${ }^{2}$ National Radio Astronomy Observatory, Socorro, NM 87801, USA \\ Received 2018 January 19; revised 2018 July 24; accepted 2018 August 10; published 2018 September 18
}

\begin{abstract}
While satellites of mid- to small-Kuiper Belt objects tend to be similar in size and brightness to their primaries, the largest Kuiper Belt objects preferentially have satellites with small fractional brightness. In the two cases where the sizes and albedos of the small faint satellites have been measured, these satellites are seen to be small icy fragments consistent with collisional formation. Here, we examine Dysnomia and Vanth, the satellites of Eris and Orcus, respectively. Using the Atacama Large Millimeter Array, we obtain the first spatially resolved observations of these systems at thermal wavelengths. Vanth is easily seen in individual images, and we find a $3.5 \sigma$ detection of Dysnomia by stacking all of the data on the known position of the satellite. We calculate a diameter for Dysnomia of $700 \pm 115 \mathrm{~km}$ and for Vanth of $475 \pm 75 \mathrm{~km}$, with albedos of $0.04_{-0.01}^{+0.02}$ and $0.08 \pm 0.02$, respectively. Both Dysnomia and Vanth are indistinguishable from typical Kuiper Belt objects of their size. Potential implications for the formation of these types of satellites are discussed.
\end{abstract}

Key words: Kuiper belt objects: individual (Eris, Orcus)

\section{Introduction}

Most of the largest objects in the Kuiper Belt are known to have one or more satellites orbiting the parent body. The majority of these satellites have a small fractional brightness compared to their parent body. Even before the discovery of any of these small satellites, models predicted that giant impacts onto differentiated bodies would preferentially form icy satellites with a small fractional mass (Canup 2005). Many of the known satellites to large Kuiper Belt objects (KBOs) appear consistent with this paradigm. In the two cases where compositional information of these small satellites is available, these satellite surfaces are known to have a high albedo and to be dominated by water ice. The small satellites of Pluto have been directly imaged by the New Horizons spacecraft and have measured albedos of 0.5-0.9 and deep water ice absorptions in the near-infrared (Weaver et al. 2016; Cook et al. 2017), while the satellites of Haumea show deep water ice absorption (Barkume et al. 2006; Fraser \& Brown 2009), and dynamical modeling strongly suggests low mass and thus high albedo (Ragozzine \& Brown 2009).

Little is known about the size or albedo of other satellites around large KBOs owing to the difficulty of resolving the satellites at anything other than optical or near-infrared wavelengths. The recently improved capability of the Atacama Large Millimeter Array (ALMA) to obtain spatial resolutions of 10s of milliarcseconds, however, allows us to now measure thermal emission directly from KBO satellites. Here, we use spatially resolved observations from ALMA to examine the size and albedo of two satellite systems: Eris-Dysnomia and Orcus-Vanth. Dysnomia, with a fractional brightness of $0.2 \%$ that of Eris (Brown \& Schaller 2007), appears to fit the paradigm of small, icy, collisionally induced satellites surrounding all of the largest known dwarf planets (Brown et al. 2006; Parker et al. 2016; Kiss et al. 2017). A closer look at the system, however, makes this assessment less certain. The unusually high albedo of Eris of 0.97 (Sicardy 2011) makes Dysnomia's relative brightness seem artificially low. In fact, if Dysnomia has a typical small-KBO-like albedo of $\sim 5 \%$, it is as large as $630 \mathrm{~km}$. On the other hand, if Dysnomia has an icycollisional-satellite-like albedo of 0.5 or higher, it is smaller than $200 \mathrm{~km}$ in radius. This range in sizes spans a wide range of the types of satellite systems in the Kuiper Belt. Without a constraint on the size of Dysnomia, we lack a fundamental understanding of this system. A counter-example is the dwarf planet Orcus, which has a satellite-Vanth — with a fractional brightness of $9.6 \%$ and a spectrum with significantly less water ice than its primary (Brown et al. 2010). The origin of this type of dwarf planet system remains uncertain, with models from capture to collision being plausible (Ragozzine 2009).

\section{Observations}

Observations of Orcus-Vanth and Eris-Dysnomia were undertaken with the $12 \mathrm{~m}$ array of the Atacama Large Millimeter Array (ALMA). This synthesis array is a collection of radio antennas, each $12 \mathrm{~m}$ in diameter, spread out on the Altiplano in the high northern Chilean Andes. Each of the pairs of antennas acts as a two element interferometer, and the combination of all of these individual interferometers allows for the reconstruction of the full sky brightness distribution, in both dimensions (Thompson et al. 2001).

ALMA is tunable in seven discrete frequency bands, from $\sim 90$ to $\sim 950 \mathrm{GHz}$. All observations in this paper were taken in Band 7, near $350 \mathrm{GHz}$, in the "continuum" (or "TDM") mode, with the standard frequency tunings. The data is observed in four spectral windows in this mode, which for us had frequency ranges: $335.5-337.5 \mathrm{GHz} ; 337.5-339.5 \mathrm{GHz} ; 347.5-349.5 \mathrm{GHz}$; and 349.5-351.5 GHz. In the final data analysis, we average over the entire frequency range in both bands and use $345 \mathrm{GHz}$ as the effective frequency in our modeling. All of these observations are in dual-linear polarization; in the end, we combine these into a measurement of the total intensity.

Table 1 shows the observational circumstances of our data. The Eris-Dysnomia system was observed in 2015 November and December; The Orcus-Vanth system was observed in 2016 October and November. Initial calibration of the data was provided by the ALMA observatory and is done in the CASA 
Table 1

Observing Dates, Geometries, and Flux Densities

\begin{tabular}{|c|c|c|c|c|}
\hline Bodies & $\begin{array}{l}\text { Date/Time } \\
\text { (UTC) }\end{array}$ & $\begin{array}{l}\text { Distance } \\
\text { (au) }\end{array}$ & $\begin{array}{l}\text { Primary f.d. } \\
\qquad(\mathrm{mJy})\end{array}$ & $\begin{array}{l}\text { Secondary f.d. } \\
\text { (mJy) }\end{array}$ \\
\hline Orcus-Vanth & 2016 Oct $11 / 11: 02-12: 16$ & 48.8 & $1.160 \pm 0.030$ & $0.310 \pm 0.030$ \\
\hline Orcus-Vanth & 2016 Oct 13/09:54-11:08 & 48.8 & $1.120 \pm 0.060$ & $0.270 \pm 0.060$ \\
\hline Orcus-Vanth & 2016 Oct $15 / 11: 54-12: 59$ & 48.7 & $1.180 \pm 0.040$ & $0.370 \pm 0.040$ \\
\hline Orcus-Vanth & 2016 Nov 7/09:30-10:40 & 48.4 & $1.170 \pm 0.030$ & $0.400 \pm 0.030$ \\
\hline Eris-Dysnomia & 2015 Nov 09/03:25-04:35 & 95.4 & $0.803 \pm 0.076$ & $\cdots$ \\
\hline Eris-Dysnomia & 2015 Nov 13/02:50-04:00 & 95.4 & $0.893 \pm 0.071$ & $\cdots$ \\
\hline Eris-Dysnomia & 2015 Dec 04/01:15-02:25 & 95.7 & $0.825 \pm 0.080$ & $\cdots$ \\
\hline
\end{tabular}

reduction package via the ALMA pipeline (Muders et al. 2014). After the initial calibration, the data product was a set of visibilities for each of the observing dates.

At this point, we exported the data from CASA and continued the data reduction in the AIPS package. Because the primary purpose of the observations was to perform astrometry of the two systems, the observations were taken using high-resolution configurations of ALMA-with resolutions as fine as 15 mas. For the purposes of this paper, we are not concerned with such high resolution but rather simply enough resolution to distinguish the primary from the satellite. Because of this, we created images using weighting of the data, which sacrifices resolution for sensitivity (so-called "natural weighting"). The resulting images are shown in Figures 1 and 2 .

The final step of the data analysis was to estimate the observed flux density for the primary and satellite for each observation. For each image, we obtained the values in a number of ways, to check for consistency: flux density in the image; flux density in the CLEAN components; fitting a Gaussian in the image; and fitting the visibilities directly. We found relatively good agreement for all of these techniques. We note that for the visibility fits, we used point sources for all but Eris, where ALMA does slightly resolve the body. For Eris, we used a fit of a slightly limb-darkened disk, with radius of $1163 \mathrm{~km}$ (Sicardy 2011). We take the visibility fit value as the best value, as it avoids the biases of the image plane (Greisen 2004).

There is one final correction that must be made to the flux densities; a correction for atmospheric decorrelation. For interferometric observations, the Earth's atmosphere causes phase fluctuations in the measured data that will result in a net reduction in the measured flux density (Thompson et al. 2001). In theory, and under good atmospheric conditions, normal calibration will account for this decorrelation in terms of the overall flux density scale, but image plane effects will still persist (source broadening, for instance). In normal ALMA observations, decorrelation is only a minor effect, because observations are scheduled when atmospheric conditions are good for the frequency being observed. However, our observations were done with specific constraints-namely that they had to be done in a particular (high-resolution) configuration, and that they had to be done with particular time separations, in order to facilitate the astrometry. Because of these constraints, our observations were not done under optimal conditions in all cases. Fortunately, the way that astrometric observations are done with ALMA provides a convenient method for correcting for the decorrelation. Along with normal
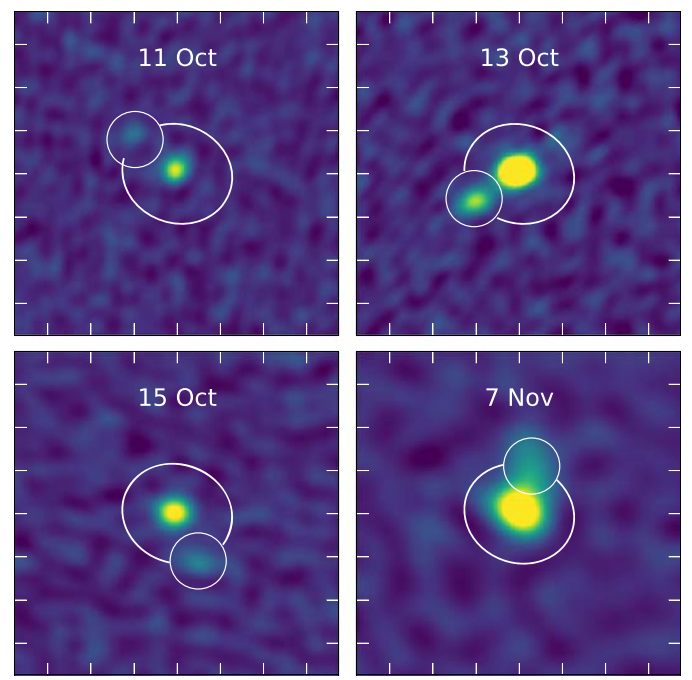

Figure 1. ALMA observations of the Orcus-Vanth system. The images are centered on Orcus, and the predicted position of Vanth is circled. Tick marks in the images are 200 mas. Vanth is clearly detected even in the lower-resolution November 7 data.
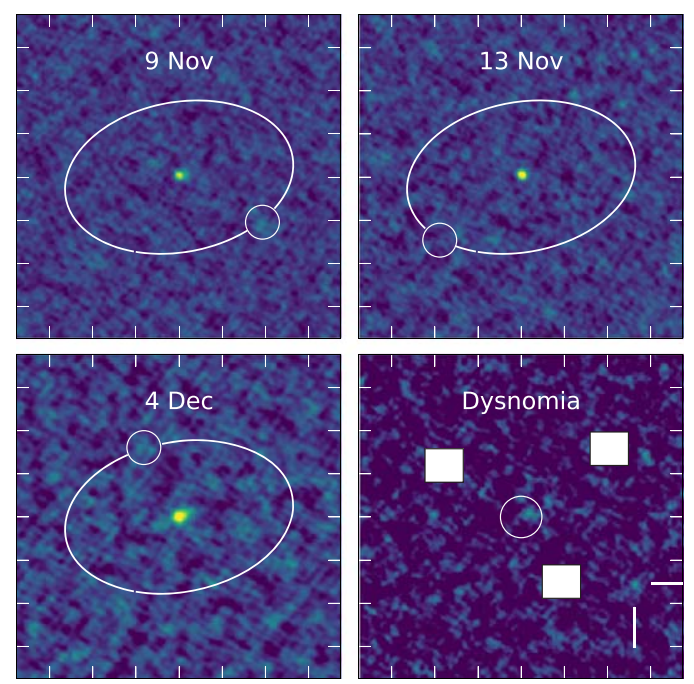

Figure 2. ALMA observations of the Eris-Dysnomia system. The images are centered on Eris, and the predicted orbit of Dysnomia is shown. Predicted positions of Dysnomia based on contemporary HST observations are circled. In the bottom right panel, the three images are shifted and stacked at the position of Dysnomia, yielding a $3.5 \sigma$ detection of a source at the position of the satellite. The three positions of the shifted image of Eris are masked with white boxes. In the full frame, only one other detection as significant (marked with cross hairs in the lower right corner of the image) is seen. 
calibrations, astrometric "check sources" are observed. These check sources are point sources with well-known astrometric positions. Because they are observed with the same time cadence as our target sources, and because they are relatively strong, self-calibration (Cornwell \& Fomalont 1999) can be used to estimate how much the flux density of these check sources changes from what the original calibration indicates. We used one of the check sources in each of the observations to measure the magnitude of this effect and applied it to our final estimate of flux densities. We note that this correction was typically small for the Eris-Dysnomia observations, but much larger for some of the Orcus-Vanth observations. Table 1 shows the final fitted flux densities, including all corrections, for all of our observations.

\section{Orcus-Vanth}

A secondary source approximately 250 mas away from Orcus is clearly visible in three of the four ALMA images, and a two-Gaussian fit also picks one out in the lower-resolution image from 2016 November 7. Using published Vanth orbital elements (Brown et al. 2010; Carry et al. 2011), we find that these detections are all along the orbital path of Vanth and consistent with the predicted position if the mean anomaly of Vanth is increased by $11^{\circ}$, well within the current uncertainties. Flux densities measured for Orcus and Vanth are shown in Table 1.

We use the measured thermal emission to determine the sizes of Orcus and Vanth using the techniques detailed in Brown \& Butler (2017, hereafter BB17). In our analysis, we use a standard thermal model to calculate the thermal emission expected from a distant body. In this model, the free parameters are bolometric emissivity, phase integral, albedo, diameter, and a beaming parameter to account for the combined effects of viewing geometry and surface thermal properties. For the emissivity and phase integral, we use typical KBO assumptions: we constrain emissivity to be between 0.8 and 1.0 , as argued in BB17, and we use the Brucker et al. (2009) empirical fit of phase integral to albedo with an allowed $50 \%$ variation from these values. Orcus and Vanth are not constrained to have any identical parameters.

We use the Markov Chain Monte Carlo scheme described in BB17 to explore the best-fit parameters and their uncertainties. We fit the unresolved Orcus+Vanth Spitzer 24 and $71 \mu \mathrm{m}$ fluxes (Stansberry et al. 2008), the unresolved Herschel 70, 100, and $350 \mu \mathrm{m}$ flux (Fornasier et al. 2013), and the new resolved ALMA data. We assume an $850 \mu \mathrm{m}$ emissivity of 0.685 , as derived in BB17, consistent with the value also found in Lellouch et al. (2017). Figure 3 shows a collection of 30 random samples from the MCMC ensemble. The fit of the model to the data is excellent.

The marginalized distributions for the size and albedo of both bodies are nearly Gaussian, so we report the median and 16 th and 84th percentiles as our $1 \sigma$ error range. We find that Orcus has a diameter of $910_{-40}^{+50} \mathrm{~km}$ and an albedo of $0.23 \pm 0.02$, while Vanth has a diameter of $475 \pm 75 \mathrm{~km}$ and an albedo of $0.08 \pm 0.02$. Vanth is approximately half of the diameter of Orcus, with an albedo approximately three times smaller. These results put Vanth within the range of typical KBO albedos for objects of this size.

Without a knowledge of the density of Vanth, the mass ratio of the system is unclear. For plausible densities from $0.8 \mathrm{~g} \mathrm{~cm}^{-3}$ (the typical density for a $\sim 500 \mathrm{~km}$ object) up to

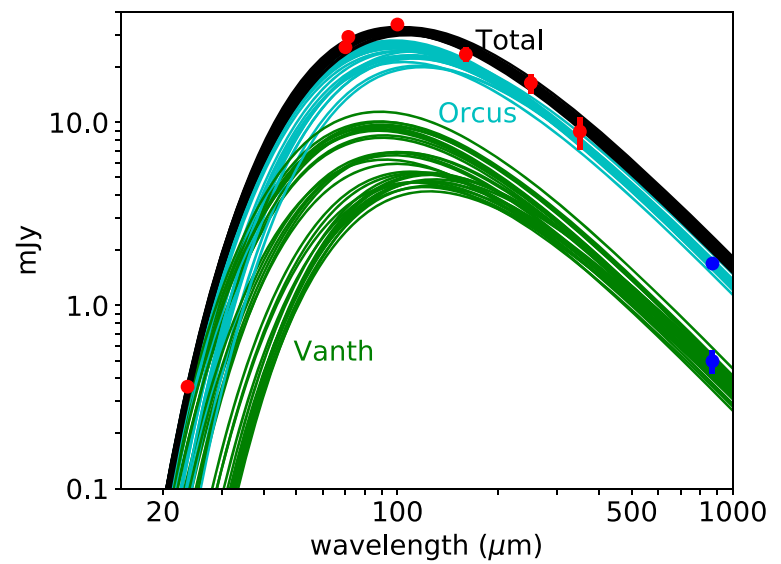

Figure 3. A collection of 30 random samples from the MCMC ensemble compared to data from the Orcus-Vanth system. The red points are unresolved data from Spitzer and Herschel, while the blue points are the resolved data points from ALMA. The ALMA data have been scaled by the inverse of the assumed emissivity of 0.685 so that they appear at the equivalent emissivity of unity locations.

Table 2

Positions of Dysnomia

\begin{tabular}{lrcl}
\hline \hline JD & \multicolumn{1}{c}{$\Delta$ R.A. } & $\begin{array}{c}\Delta \text { Decl. } \\
\text { mas }\end{array}$ & Observatory \\
& \multicolumn{1}{c}{ mas } & $-226 \pm 1$ & HST (measured) \\
\hline 2457051.699 & $-347 \pm 2$ & $-325 \pm 2$ & $H S T$ (measured) \\
2457054.950 & $282 \pm 3$ & & \\
& & $-210.3 \pm 1.0$ & ALMA (predicted) \\
2457335.667 & $-383.2 \pm 1.4$ & $-291.5 \pm 1.7$ & ALMA (predicted) \\
2457339.641 & $379.5 \pm 1.5$ & $322.8 \pm 1.0$ & ALMA (predicted) \\
2457360.576 & $159.8 \pm 1.7$ & & \\
\hline
\end{tabular}

$1.4 \mathrm{~g} \mathrm{~cm}^{-3}$ (the system density if Orcus and Vanth have identical densities), the mass ratio ranges between 5 and 20, while the density of Orcus ranges from $1.4 \pm 0.2$ to $2.0 \pm 0.3 \mathrm{~g} \mathrm{~cm}^{-3}$. Clearly, determining the density of Vanth is critical to understanding how the Orcus-Vanth systems fits into our understanding of large KBOs and their densities.

\section{Eris-Dysnomia}

No obvious detections of Dysnomia appear in the data. If we knew the predicted position of Dysnomia with respect to Eris, however, we could make a more stringent determination. The last published orbital elements of Dysnomia (Brown \& Schaller 2007) have a $30^{\circ}$ phase uncertainty by the time of these observations, so are not sufficient for providing predictions. We thus use archival observations obtained using WF3 on the Hubble Space Telescope in 2015 January to update the orbital elements of Dysnomia and precisely predict its position at the time of the ALMA observations 9 months later. Astrometric offsets between Eris and Dysnomia for the times of observation are determined using the methods detailed in Brown \& Schaller (2007). Table 2 shows the relative positions of Dysnomia at the times of the HST observations.

We calculate the new orbit of Dysnomia using the methods described in Brown \& Schaller (2007) with the updated improvements using a Markov Chain Monte Carlo scheme as described in Brown (2013). The orbit is in agreement with the previous results but with improved uncertainties. The orbit continues to be consistent with being circular, with a $1 \sigma$ upper limit to the eccentricity of 0.004 , so for the final fit we constrain 


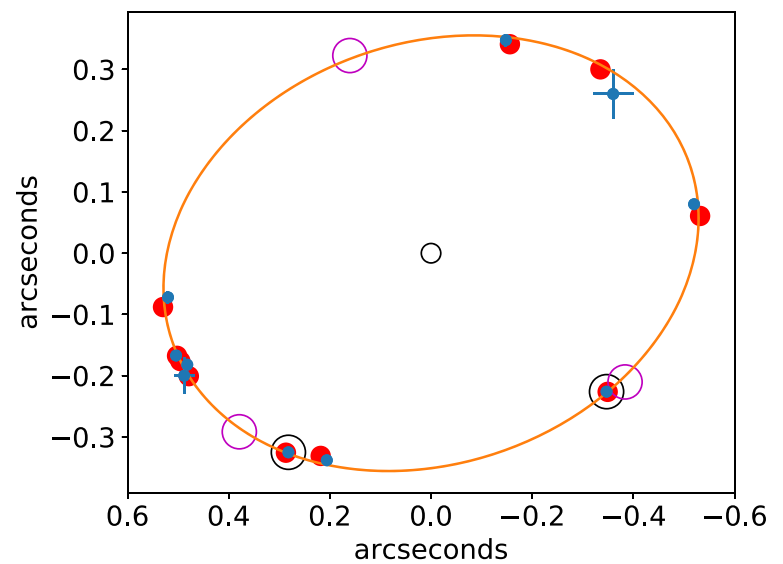

Figure 4. A fit to the orbit of Dysnomia. Blue dots with error bars are the observations, while their best-fit locations are show as red dots. The two new data points added in this analysis are circled in blue. The large unfilled cyan circles are the predicted locations of Dysnomia at the times of the ALMA observations.

the orbit to be purely circular (Figure 4). Updated orbital elements are provided in Table 3.

The new orbit fit allows us to predict the locations of Dysnomia at the times of the ALMA observation nine months later to within $\sim 2$ mas, a fraction of the ALMA beam size. Additional astrometric uncertainty of order $\sim 10$ mas (onefourth of the angular diameter of Eris) arises because of the potential offset between the center-of-light and center-of-mass of Eris (if, for example, Eris has a warmer pole observed obliquely). We show these predicted locations in Figures 2 and 4. A flux density enhancement occurs at the predicted location in at least two of the three observations. To increase the signal-to-noise of a potential Dysnomia detection, we shift all three images to be centered on the predicted position of Dysnomia and sum them (Figure 2). A source with a flux density of $0.13 \pm 0.03 \mathrm{mJy}$ appears 16 mas from the expected position, smaller than the 30-50 mas resolution element of the data and within the range of expected astrometric uncertainties for such a low signal-to-noise source.

While we formally have a $4.2 \sigma$ detection of a potential source near the position of Dysnomia, we assess the true significance of this potential detection by performing an experiment where we shift each image by random amounts and determine the maximum flux density within 30 mas of this arbitrary position. We find values as high as the $0.13 \mathrm{mJy}$ of Dysnomia only $0.02 \%$ of the time, corresponding to a $3.5 \sigma$ likelihood that the detection is indeed Dysnomia. As an additional check, we reexamine the combined image and find that in the 2.75 square arcsecond field we only see one other source as strong as the potential Dysnomia detection. Our field consists of approximately 1400 resolution elements, so we should expect to randomly detect a $3.5 \sigma$ source $33 \%$ of time, consistent with the observation. While the detection of Dysnomia is weak, we can find no reason to discount its reality. We proceed on the assumption that we have indeed detected Dysnomia.

We model the emission from Eris and Dysnomia using an identical technique as previously used for Orcus-Vanth. We assume the occultation-derived circular diameter of Eris of $2326 \pm 12 \mathrm{~km}$ (Sicardy 2011) and have as the free parameters in our model fitting the beaming parameter of Eris and the diameter, albedo, and beaming parameter of Dysnomia. We
Table 3

Orbital Elements of Dysnomia

\begin{tabular}{lcc}
\hline \hline Semimajor Axis & $37460 \pm 80$ & $\mathrm{~km}$ \\
Inclination & $61.1 \pm 0.3$ & $\mathrm{deg}$ \\
Period & $15.78586 \pm 0.00008$ & days \\
Eccentricity & $<0.004$ & \\
Long. Ascending Node & $139.6 \pm 0.2$ & $\mathrm{deg}$ \\
Mean Anomaly & $273.2 \pm 0.02$ & $\mathrm{deg}$ \\
Epoch (JD, defined) & 2457054.95 & $\ldots$ \\
\hline
\end{tabular}

Note. Relative to J2000 ecliptic.

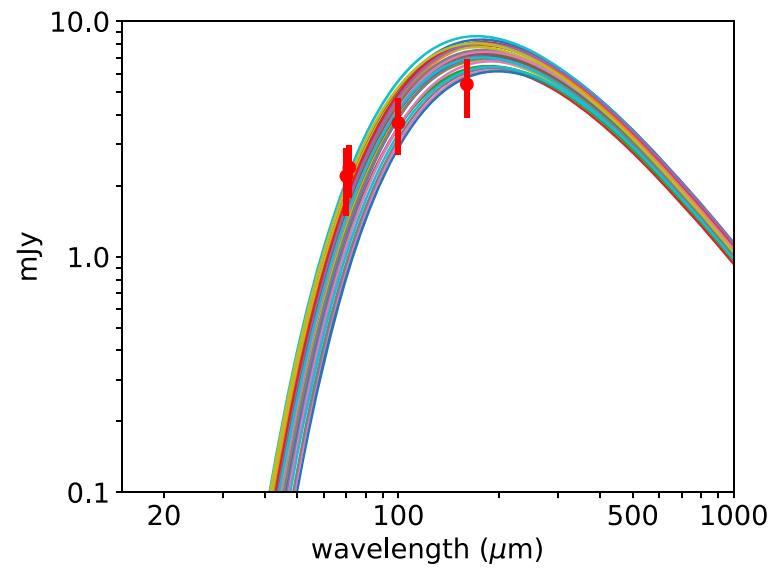

Figure 5. A collection of 30 random samples from the MCMC ensemble compared to data from the Eris-Dysnomia system. We model only a single body and the unresolved measurements from Herschel. While the fit does not statistically support the existence of a second body in the system, it is clear that a smaller, darker (thus warmer) body could improve the fit to the data.

again constrain the bolometric emissivity and Bond albedo as above.

We first investigate if there is any evidence for emission from Dysnomia from previous, unresolved data. We use data at $71 \mu \mathrm{m}$ from the Spitzer Space Telescope (Stansberry et al. 2008) and data at 70, 100, and $150 \mu \mathrm{m}$ from the Herschel Space Telescope (Santos-Sanz et al. 2012). The marginalized distribution for the diameter of Dysnomia gives a $1 \sigma$ upper limit of $810 \mathrm{~km}$ and a corresponding $1 \sigma$ lower limit for albedo of 0.03 . The unresolved data provide essentially no information on the parameters of Dysnomia. Nonetheless, if no Dysnomia is included in the fit, the flux of the shortest wavelength data is under-predicted, suggesting the possibility that a large dark body could be present in the system (Figure 5).

We next fit the resolved ALMA data together with the previous unresolved data. We continue to use our earlierderived $850 \mu \mathrm{m}$ emissivity of 0.685 for Dysnomia, assuming it is a typical KBO. For Eris, which has a significantly different surface composition, we consider ALMA observations of Pluto (Butler et al. 2015), which suggest significantly lower brightness temperature. However, it is difficult to separate the effects of emissivity and cold atmospherically buffered $\mathrm{N}_{2}$ ice. As $\mathrm{N}_{2}$ ice at the temperature of Eris has significantly lower volatility, we assume that ice effects are negligible and instead use the same canonical 0.685 value for emissivity. Figure 6 shows 30 samples from the MCMC ensemble to the thermal data. We find that Dysnomia has a diameter of $700 \pm 115 \mathrm{~km}$ with an albedo of $0.04_{-0.01}^{+0.02}$. Dysnomia's size and albedo are consistent with those of typical mid-sized KBOs, but the albedo is nearly 25 times smaller than the extremely bright Eris. Allowing the 


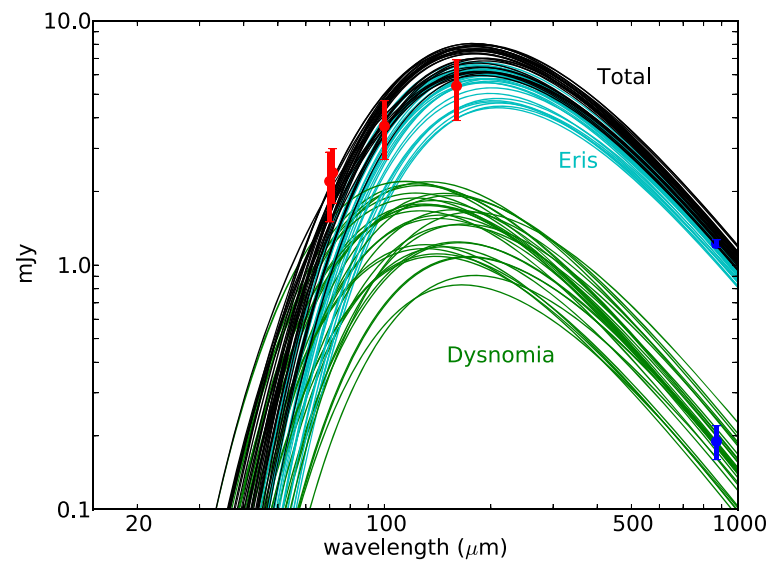

Figure 6. A collection of 30 random samples from the MCMC ensemble compared to data from the Eris-Dysnomia system. Including the resolved ALMA data shows the presence of a mid-sized dark Dysnomia, which somewhat improves the fit to the shorter wavelength data where Dysnomia's contribution is more significant.

density of Dysnomia to range from as low as $0.8 \mathrm{~g} \mathrm{~cm}^{-3}$ to as high as being equal to that of Eris gives a range of system mass ratios between 37 and 115, again a large possible range.

\section{Discussion}

Neither Vanth, the satellite of Orcus, nor Dysnomia, the satellite of Eris, fits the paradigm of the type of small icy collisionally derived satellite predicted by the models of Canup (2005). Such satellites form out of the icy disk surrounding the parent in the aftermath of the collision and would be expected to be nearly pure water ice, like the satellites of Haumea and the small satellites of Pluto appear to be. Indeed, both Dysnomia and Vanth have the low albedos expected for typical KBOs of their size (Stansberry et al. 2008). We consider possible formation mechanisms below.

Smaller multiple-KBO systems tend to be binaries with similar-sized components (Noll et al. 2008). These are thought to either be formed through dynamical-friction-assisted capture (Goldreich et al. 2002) or to have initially formed as a pair (Nesvorný et al. 2010). Little exploration has been done on the range of possible satellite parameters that could ensue from such mechanisms, but they are generally thought to be relevant to small KBO pairs. There is no reason to expect that while mid-sized KBOs have a modest satellite fraction, the largest KBOs would preferentially have satellites owing to these same mechanisms.

Charon, the large satellite of Pluto, appears to have formed from a grazing giant impact, which then left the proto-Charon largely intact, but with a low enough energy to remain bound (Canup 2005). In simulations, collisions of undifferentiated bodies mostly yielded a satellite whose composition was unchanged from the initial impactor. The Orcus-Vanth system is plausibly explained by such a scenario. With a mass ratio between 5 and 20, Orcus-Vanth appears to be a good candidate for an analog to the Pluto-Charon system (with a mass ratio of 8 ), with the exception that we detect no small icy analogs of the small Pluto satellite. Brown (2008) places a brightness ratio limit of $0.1 \%$ for any more distant undiscovered objects in the Orcus system. For an icy albedo of $0.5-1.0$, this limit corresponds to objects with a diameter of $15-20 \mathrm{~km}$, smaller than at least two of the small satellites of Pluto.
The Eris-Dysnomia system is unlike any other known in the Kuiper Belt. With a mass ratio between 37 and 115, Dysnomia appears intermediate between satellites such as Charon and Nyx and Hydra (with mass ratios greater than $10^{5}$; Brozović et al. 2015) Hi' iaka, the larger satellite of Haumea (with a mass ratio of $\sim 200$; Ragozzine \& Brown 2009). But an albedo of $\sim 0.04$ clearly shows that Dysnomia is not a reassembled product of an icy disk. We suggest two alternatives. First, it is possible that our reported detection of Dysnomia is erroneous. While we showed that the probability of a spurious detection at the predicted combined location of Dysnomia is low, robust detection at multiple locations is desired. Second, if the detection of Dysnomia is real, as the statistics suggest, our understanding of Kuiper Belt satellite formation mechanisms is clearly inadequate, and the range of possible satellite formation outcomes is larger than currently thought.

While large KBOs have a high fraction of satellites, a general understanding of the diverse possible formation mechanisms for these satellites is lacking. It is generally believed that giant impacts are responsible for the satellites of these KBOs, but simulations have only attempted to explain individual systems (Canup 2005, 2011; Leinhardt et al. 2010), and no comprehensive study has explored a wide range of possible outcomes. Barr \& Schwamb (2016) have suggested a general paradigm where collisions are either of the Charonforming type or of the icy-small-fragment type, but it is not clear if this paradigm can be reconciled with the implication that Dysnomia appears not to have formed from a post-impact icy disk of material. As understanding of these satellite systems likely provides insights into populations and collisions in the early outer solar system, emphasis should be placed on both the theoretical and observational exploration of these objects.

This paper makes use of the following ALMA data: ADS/JAO. ALMA\#2016.1.00830.S, ADS/JAO.ALMA\#2015.1.00810.S. ALMA is a partnership of ESO (representing its member states), NSF (USA) and NINS (Japan), together with NRC (Canada), MOST and ASIAA (Taiwan), and KASI (Republic of Korea), in cooperation with the Republic of Chile. The Joint ALMA Observatory is operated by ESO, AUI/NRAO and NAOJ. The National Radio Astronomy Observatory is a facility of the National Science Foundation operated under cooperative agreement by Associated Universities, Inc.

\section{ORCID iDs}

Michael E. Brown (iD https://orcid.org/0000-0002-8255-0545

\section{References}

Barkume, K. M., Brown, M. E., \& Schaller, E. L. 2006, ApJL, 640, L87 Barr, A. C., \& Schwamb, M. E. 2016, MNRAS, 460, 1542

Brown, M. E. 2013, ApJL, 778, L34

Brown, M. E., \& Butler, B. J. 2017, AJ, 154, 19

Brown, M. E., Ragozzine, D., Stansberry, J., \& Fraser, W. C. 2010, AJ, 139,2700

Brown, M. E., \& Schaller, E. L. 2007, Sci, 316, 1585

Brown, M. E., van Dam, M. A., Bouchez, A. H., et al. 2006, ApJL, 639, L43 Brown, M. E. 2008, in The Solar System Beyond Neptune, ed. M. A. Barucci (Tucson, AZ: Univ. Arizona Press), 335

Brozović, M., Showalter, M. R., Jacobson, R. A., \& Buie, M. W. 2015, Icar, 246, 317

Brucker, M. J., Grundy, W. M., Stansberry, J. A., et al. 2009, Icar, 201, 284

Butler, B. J., Gurwell, M., Lellouch, E., et al. 2015, AAS/DPS Meeting, 47, 210.04 
Canup, R. M. 2005, Sci, 307, 546

Canup, R. M. 2011, AJ, 141, 35

Carry, B., Hestroffer, D., DeMeo, F. E., et al. 2011, A\&A, 534, A115

Cook, J. C., Binzel, R. P., Cruikshank, D. P., et al. New Horizons Composition Theme Team, \& Ralph Instrument Team 2017, LPSC, 48, 2478

Cornwell, T., \& Fomalont, E. B. 1999, in ASP Conf. Ser. 180, Synthesis Imaging in Radio Astronomy II, ed. G. B. Taylor, C. L. Carilli, \& R. A. Perley (San Francisco, CA: ASP), 187

Fornasier, S., Lellouch, E., Müller, T., et al. 2013, A\&A, 555, A15

Fraser, W. C., \& Brown, M. E. 2009, ApJL, 695, L1

Goldreich, P., Lithwick, Y., \& Sari, R. 2002, Natur, 420, 643

Greisen, E. W. 2004, in ASP Conf. Ser. 281, Astronomical Data Analysis Software and Systems XI, ed. D. A. Bohlender, D. Durand, \& T. H. Handley (San Francisco, CA: ASP), 260

Kiss, C., Marton, G., Farkas-Takács, A., et al. 2017, ApJL, 838, L1

Leinhardt, Z. M., Marcus, R. A., \& Stewart, S. T. 2010, ApJ, 714, 1789

Lellouch, E., Moreno, R., Müller, T., et al. 2017, A\&A, 608, A45
Muders, D., Wyrowski, F., Lightfoot, J., et al. 2014, in ASP Conf. Ser. 485 , Astronomical Data Analysis Software and Systems XXIII, ed. N. Manset \& P. Forshay (San Francisco, CA: ASP), 383

Nesvorný, D., Youdin, A. N., \& Richardson, D. C. 2010, AJ, 140, 785

Noll, K. S., Grundy, W. M., Chiang, E. I., Margot, J.-L., \& Kern, S. D. 2008, in The Solar System Beyond Neptune, ed. M. A. Barucci (Tucson, AZ: Univ. Arizona Press), 345

Parker, A. H., Buie, M. W., Grundy, W. M., \& Noll, K. S. 2016, ApJL, 825, L9

Ragozzine, D., \& Brown, M. E. 2009, AJ, 137, 4766

Ragozzine, D. A. 2009, PhD thesis, California Institute of Technology

Santos-Sanz, P., Lellouch, E., Fornasier, S., et al. 2012, A\&A, 541, A92

Sicardy, B., Ortiz, J. L., Assafin, M., et al. 2011, Natur, 478, 493

Stansberry, J., Grundy, W., Brown, M., et al. 2008, in The Solar System Beyond Neptune, ed. M. A. Barucci (Tucson, AZ: Univ. Arizona Press), 161

Thompson, A. R., Moran, J. M., \& Swenson, G. W. 2001, Interferometry and Synthesis in Radio Astronomy (2nd ed.; New York: Wiley-Interscience)

Weaver, H. A., Buie, M. W., Buratti, B. J., et al. 2016, Sci, 351, aae0030 